\title{
El diseño, una responsabilidad social en el uso adecuado de diseños étnicos
}

\section{Design, a social responsibility in the proper use of ethnic designs}

Verinha Margarita Domínguez Vela Universidad Autónoma de Ciudad Juárez (UACJ)

veridom.v@gmail.com

Ciudad Juárez, Chihuahua, México

ORCID: 0000-0001-9148-4781
Erika Anastacia Rogel Villalba Universidad Autónoma de Ciudad Juárez (UACJ) erogel@uacj.mx Ciudad Juárez, Chihuahua, México ORCID: 0000-0002-0219-0173

Recibido: 14 de noviembre de 2018 Aprobado: 12 de diciembre de 2018 Publicado: 01 de enero de 2019

\section{s) Resumen}

El presente texto plantea una problemática sobre el papel de los diseñadores y las marcas que basan sus productos de diseño en la iconografía de grupos étnicos, aplicados sin retribuirles el debido reconocimiento de autoría. Se pretende a través de este, remarcar la responsabilidad que debe mantenerse entre el diseño y la cultura. Así como, la ética del diseñador al considerar los trabajos de estos grupos a partir de una perspectiva sociocultural. Ya que, de manera sustancial son estos los diseños que se encuentran sin la protección legal a nivel nacional e internacional. El estudio tiene como objetivo proponer un decálogo para los diseñadores a través de una investigación de corte cualitativo. Este trabajo va orientado a la reflexión ética, la responsabilidad, los principios y los valores fundamentales en la formación de los estudiantes de diseño.

Palabras clave: cultura, diseño, ética, etnias, responsabilidad social.

\section{Abstract}

This text raises a problem about the role of designers and brands that base their design products on the iconography of ethnic groups, applied without giving them due recognition of authorship. The aim is to emphasize the responsibility that must be maintained between design and culture. As well as, the ethics of the designer when considering the works of these groups from a sociocultural perspective. Since, in a substantial way, these are the designs that are found without legal protection at a national and international level. The research aims to propose a decalogue for designers through a qualitative research. This work is oriented towards ethical reflection, responsibility, principles and fundamental values in the training of design students.

Keywords: culture, design, ethics, ethnicity, social responsibility. 


\title{
Introducción
}

\begin{abstract}
$M$ pesar de la existencia del Instituto Mexicano de la Propiedad Industrial (IMPI), institución para el reconocimiento legal de la propiedad intelectual, como del Instituto Nacional de Antropología e Historia (INAH), existe un escaso apoyo por parte del gobierno mexicano en el registro de diseños de patrones e iconografías étnicas. En general, los diseñadores realizan usos de estos recursos étnicos con problemas en materia legal. Situación debida en gran medida, a la falta de protección legal de estos diseños. Por otro lado, esta problemática también puede darse a causa de la desinformación sobre los usos lícitos de estos diseños, el desconocimiento de la propiedad intelectual o por la falta de ética. Estos factores conllevan a prácticas inadecuadas en el diseño. Sin embargo, la responsabilidad ética y profesional del diseñador que quiera hacer algún uso de los iconos o la simbología pertenecientede estas culturas representativas, debería partir del reconocimiento intelectual para acoger estos diseños pertenecientes a estas culturas, en este o en cualquier otro país. Esto implica, la parte moral sobre la paternidad de los diseños y la debida retribución económica por la explotación de esta creación.
\end{abstract}

El enfoque responsable del diseñador, como creador y consumidor, en el diseño y la producción de la sociedad actual es, llegar a ser el enlace del cambio hacia un nuevo paradigma. Esto es, un vínculo para el consumo de diseño de manera sustentable. Hoy por hoy, el diseñador ocupa un rol clave en el desarrollo de estrategias creativas y sustentables, pero cabría preguntarse si actúa de forma responsable y ética como consumidor y creador; parece una pregunta fácil con una respuesta aún más sencilla. Sin embargo, la responsabilidad del diseñador lo ha llevado a contemplar y aplicar estrategias que fomenten estos valores.

Es por ello que, el diseñador debe tener una participación activa en el ámbito social $y$, esto no se refiere únicamente a la producción de una publicidad exitosa, en la cual, uno de los principales objetivos es el de ocuparse por corresponder a los parámetros estéticos y funcionales solicitados por el cliente. Le concierne del mismo modo, conducirse bajo un código ético, implícito en originalidad y autoría del diseño, más tratándose de los principios ligados a la honradez, la lealtad, la honestidad, la honorabilidad y la justicia. Por tanto, todo individuo sea diseñador, cliente o usuario, deberá manejarse respetuoso y profesional en el uso de la creación e información del producto diseñado, en armonía con los reglamentos legales al respecto (Huidobro, 2005). En el caso particular del diseño gráfico, desde su surgimiento como disciplina, ha evolucionado constantemente gracias a los medios masivos de comunicación 
y la publicidad. De modo que, los diseñadores gráficos deberán estar capacitados en diversos temas culturales y legales, así como lo hace en el manejo de herramientas para la producción con el uso de tecnología especializada. Puesto que son muchas las áreas en las que puede intervenir, incluso cuando el problema es demasiado complejo y requiere de una formación multi y transdisciplinaria.

De acuerdo con el documento publicado por López (2013), titulado «Relación entre el diseño gráfico y las leyes», nos encontramos en un campo laboral en el cual la competencia desleal aumenta cada vez más, afectando a los diseñadores. Situación que se ve agudizada por los acelerados procesos de producción que no permiten un trabajo de diseño fundamentado en la originalidad, pues este desarrollo creativo conlleva un tiempo considerable en su gestión. Es en este contexto de la premura donde se puede sucumbir al hacer uso de material no original y llegar a cometer plagio. Se puede mencionar una forma sutil como es aquella que consiste en copiar un producto y hacerlo pasar como una idea original difundida a nivel local, nacional o internacional, usualmente a un costo bajo. Otro caso es, el de diseñadores de renombradas marcas que han llegado a copiar de manera exacta la iconografía de las etnias para plasmarla sobre sus productos de diseño, limitándose a decir que ayudan a su difusión cultural, cuando lo que se ha realizado es una práctica ilícita de esta propiedad.

La carrera de Diseño Gráfico en México es relativamente nueva, por lo que hasta ahora no existe un código de ética formalmente establecido. La Universidad Nacional Autónoma de México (UNAM) propuso un código ético a partir del año 2010. Este es un conjunto de principios compartidos, a través de los cuales promover y fomentar de manera integral el respeto a la profesión, los colegas, los clientes, el consumidor, la ecología y la sociedad. Dicho documento está compuesto por siete secciones en las que se establecen los principios con los cuales ha de dirigirse la comunidad de diseñadores en cuanto al desarrollo y ejercicio de la profesión, dentro y fuera del campo laboral. También se establecen normas de responsabilidad ética hacia la sociedad y el medio en el que se vive. Estos códigos se relacionan con el cliente, el beneficio del usuario, la responsabilidad hacia la comunidad y a los diseñadores, la protección del ecosistema, la formación de la identidad cultural y el mejoramiento de la profesión.

Es necesario comprender que cuando se habla sobre la identidad cultural en este código, se enfatiza el deber de enriquecerla y priorizarla para promoverla e incorporarla en las costumbres y tradiciones culturales del diseño mexicano. La norma indica textualmente partir del artículo 5, sección VI:

Definir la identidad de los grupos culturales. Artículo 6. Difundir las costumbres y tradiciones mexicanas más allá de la propia comunidad, para que siempre estén presentes y no pasen al olvido. Artículo 
7. Darle mayor valor a los diseños y propuestas que contengan un estilo propio en cuanto a la cultura mexicana, dejando de recurrir a los estilos impuestos por naciones extranjeras. Artículo 8. El profesional de diseño y comunicación visual, no realizará trabajos que denigren, tergiversen o promuevan falsas apreciaciones de la identidad cultural nacional (UNAM, 2010, 22).

Puede inferirse, de acuerdo al último apartado del texto citado que, el diseñador debe ser honesto en cuanto a su originalidad, no utilizar recursos sin el consentimiento del autor y siempre dar crédito o participación en la realización de algún diseño según corresponda. Pese a estos códigos, queda la problemática del no reconocimiento a los autores. Ya que, solo se indica en el artículo 10 de la sección VII que el diseñador y el comunicador visual deben de: «actuar conforme a las leyes vigentes en el país de su residencia, independientemente de su nacionalidad» (ídem).

Por otro lado, desde el punto de vista de Kate Fletcher (2010) y las investigaciones en el diseño de indumentaria de Sofía Marré (2012) el diseñador debe: asumir la responsabilidad tanto en el hacer-crear como en el comunicar al público el consumo de diseño de una manera ética. Por otra parte, López (2016) señala que es importante en la formación de nuevos consumidores tomar en cuenta las demandas individuales y colectivas de los diseñadores, manufactureros y consumidores.

\% La vestimenta como medio de representación de identidad sociocultural
Los estudios relacionados con la indumentaria y la moda indígena refieren a una tradición milenaria de las prendas sin considerar el mestizaje durante el período posrevolucionario. Ahora se sabe que este proceso desempeñó un papel fundamental en la confección, el diseño y evolución de las prendas indígenas desde el siglo XIX, ejemplo de ello son: las telas importadas de Europa; los tintes naturales y sintéticos; los algodones maquilados y los hilos mercerizados. Estos son algunos de los elementos que han transformado este proceso sociocultural.

Un caso que ayuda a comprender este contacto entre culturas es la elaboración del quechquémitl, de tradición precolombina. Los siglos XX y XXI no son la excepción del influjo de las tendencias marcadas por la moda en esta indumentaria. La evolución estética de esta prenda, puede señalarse a través de dos tipos de fenómenos: uno interno, propuesto desde la misma comunidad y otro externo, con un estilo actual y una tendencia foránea. Así, un diseño novedoso realizado por alguna mujer tejedora puede ser adoptado pronto por toda una comunidad y en un par de décadas, tornarse en tradición. Esto permite ver una dinámica sociocultural, no solo desde una perspectiva ritual de la vestimenta endémica e inamovible (Mendoza, 2012).

De modo que, para el análisis del uso de símbolos, iconos y diseños étnicos sobre estampados de vestimentas o artesanías, es necesario comprender 
por principio ¿por qué las marcas de moda han abusado del uso de recursos étnicos gráficos para la producción y venta de sus productos? Es de observar el fin a partir del cual se intenta explicar como medio de identidad social y cultural, para promover la representación de las culturas de cada ciudad a través de la moda, utilizando estampados en lugar de bordados sobre las telas para una producción masiva.

Al abordar el presente estudio sobre la identidad de una cultura es preciso hacer un acercamiento a la indumentaria y la moda. Debido a sus implicaciones socioculturales y su significación como imagen. La descripción de los diferentes aspectos antropológicos, sociológicos y culturales del fenómeno de la moda impide una reflexión concisa. En principio, se comprende aquí a la identidad en el modo de vestir como la expresión social de un estilo de vida. Es posible dada esta definición entender por moda, una tendencia social globalizada hacia la imitación establecida a través de las marcas del mercado, las cuales proponen un modo de vestir étnico, sin tomar en cuenta que, la moda es lo que identifica a una nación (Torres, 2014).

De manera que, integrar en la producción imágenes, símbolos, iconos debería tener implicaciones que adviertan el uso de ellas en la vida sociocultural. Desde el punto de vista histórico, el impacto social sobre la vestimenta es lo que ha dado lugar a una diversidad de estilos en la forma de la ropa a través de diferentes épocas y lugares, además, del factor climático. En su evolución se ha de notar la variación de estilos, materiales, tecnologías disponibles, códigos, posición social, migración y sobre todo las tradiciones identitarias y culturales de la sociedad (ídem).

Contrarios a la tradición identitaria y cultural de la indumentaria étnica son los productos de moda elaborados a partir de ideas de mercado, que junto con las ideas del bien común deberían contribuir a la cultura de la sociedad. Si la sociedad se expresa a través de nuevas formas de lenguaje en el campo de la moda, es necesario aprender a leerlas y a hablarlas de acuerdo a su marco cultural. Dado que, la naturaleza de este lenguaje no impone un uso correcto, sino ajustado a la realidad. Es decir, «La moda es una forma de comunicación e identificación con la sociedad» (Basail, $2006,4)$. Mientras que, desde el contexto social se involucran procesos de mercado, producción, distribución y consumo. Acorde con este, la moda es una forma particular de llevar la vestimenta, es la expresión de una determinada imagen particular:

La moda es el rostro de la sociedad, está en los monumentos del pasado y por eso nos declara su edad y los ideales, el espíritu de una época y las influencias de las distintas culturas, convirtiéndose, así en auxiliar firme de la historia (Basail, 2006, 5).

Es por ello que, la identidad cultural es un proceso en el que se utiliza y representan diversos aspectos sociales, los cuales cambian acorde al lugar y tiempo; estos permanecen intrínsecos a la cultura y la moda, 
donde surge la necesidad de los pueblos de ser redescubiertos. Así, el análisis de la moda como representación social y signo cultural, aporta conocimientos sobre la historia de la humanidad. La moda, actúa como elemento de evolución sociocultural y descubre el potencial como expresión de identidad de un país, pueblo o región, portadora de infinidad de mensajes (Torres, 2014).

Ejemplos de marcas y diseñadores que basan sus productos de moda en diseños étnicos es el de la diseñadora francesa Isabel Marant, conocida en las redes sociales por plagiar en el diseño de blusas los bordados característicos de los huipiles de la comunidad Mixe de Oaxaca. A pesar de que, la comunidad indígena ha sido creadora de esos textiles, el precio de ambas creaciones expone la importancia de la protección de este patrimonio. Debido a que, mientras la realización de una blusa tradicional puede ser consumida a un costo alrededor de los $\$ 800$, el uso ilícito de los diseños en cada blusa de Marant oscila los \$4,500 (Escobar, 2015).

Este uso ilícito fue anunciado como una creación propia por parte de Marant. A pesar del evidente plagio de los diseños de la comunidad mixe, se informó que la compañía Antik Batik comenzó un juicio para reclamar reconocimiento de los derechos sobre los diseños bordados, demandando a la diseñadora por afirmar que el diseño le pertenecía. Finalmente, esta reconoció que tomó inspiración de esta comunidad y no de la marca francesa. Sin embargo, la diseñadora no tuvo repercusiones legales debido a la falta de protección de los diseños étnicos mexicanos (ídem).

Más tarde, el presidente municipal de Santa María Tlahuitoltepec, Erasmo Díaz González, mencionó que los pobladores de la comunidad Mixe se habían enterado de la noticia a través de un medio de comunicación internacional. Estos han recibido el apoyo del Museo Textil de Oaxaca y de la Fundación Alfredo Harp Helú. De acuerdo con estas instituciones, no existe un marco legal para la protección de esta propiedad indígena. Por lo cual, se hace un llamado urgente al pueblo mexicano para exigir la creación de normas legales que protejan los derechos de una marca colectiva de las comunidades artesanales del país (ídem).

El primero de julio de 2016, a un año de lo sucedido, se presentaron autoridades de Santa María Tlahuitoltepec, Oaxaca, para dar a conocer un mensaje de reflexión sobre su cultura y su bordado por medio del Centro de Estudios de Arte Popular Ruth D. Lechuga, recién abierto, dentro del Museo Franz Mayer. Al retomar el caso, los representantes de esta población invitaron a la diseñadora francesa a reunirse con ellos para darle a conocer la vida de esta comunidad y darle a saber el verdaderouso de la blusa típica. La comunidad de Santa María Tlahuitoltepec Mixe de Oaxaca, a través de su presidente municipal, hizo el siguiente llamado:

1. Que reconozcan la blusa de Tlahuitoltepec, sus elementos y sus motivos gráficos que la componen, representan la identidad 
de la comunidad. No restringen su variación en cuanto a diseño, uso o aplicación. El patrón gráfico representa un patrimonio colectivo que recrea y representa a la cultura, por lo que no permite la posesión de un solo autor.

2. Que la diseñadora Isabel Marant Etoile retire su diseño, detenga la producción bajo su firma y repare económicamente los daños ocasionados a los afectados por el plagio.

3. Invitar a Isabel Marant Etoile a conocer a las tejedoras y artesanos que producen estas blusas en los talleres de Santa María Tlahuitoltepec desde hace cientos de años, para que conozca su proceso de elaboración y reconozca de facto su diseño de origen.

4. Invitar a la sociedad civil y a las autoridades federales nacionales e internacionales correspondientes a lograr la legitimación de la herencia del colectivo, que son también productos de auto sustento para las familias.

5. Que las autoridades actúen para informar a las comunidades sobre el debido proceso legal que deben realizar para patentar sus diseños como una marca colectiva de cada región, de lo contrario casos como este continuarán vulnerando y faltando a la integridad de la identidad cultural.

Se queda en la espera de la debida disculpa pública. Además, se insiste en la necesidad de creación, así como el uso de mecanismos de protección nacional como internacional, para la propiedad colectiva de bienes tangibles e intangibles de las comunidades y los pueblos indígenas (Fomento Cultural Banamex, 2016).

Consecuente a estas acciones es la de evitar estas situaciones como ya se ha hecho en otros contextos con marcas colectivas registradas, como la de los Navajos en Estados Unidos de América o la ley de protección de diseños étnicos aprobada en Guatemala.

Además del uso de los diseños étnicos sobre la vestimenta, también y artesanía se puede observar su empleo en la industria del calzado. A saber, han inspirado a marcas como New Balance, Vans y Nike, quienes han sacado a la venta una colección basada en el diseño étnico huichol y tarahumara, plasmados como obras de arte coloridas y extravagantes sobre tenis de diseñadores internacionales como Christian Louboutin y Dione.

Fue en el mes de agosto del año 2013 cuando la revista Esquire Latinoamérica, publicó que la marca Nike había lanzado una nueva línea de tenis: Free Flyknit, inspirados en los tarahumaras o rarámuris y de la integración de personas de esta comunidad en la nueva publicidad de la 
marca deportiva. Brindándoles de esta manera el crédito en la campaña publicitaria por este producto basado en su cultura. El nombre rarámuri refiere a pies ligeros o corredores a pie, hombres cuya tradición de correr descalzos fue la que inspiró a la marca en la creación de la colección de este calzado, la cual es ligera y flexible, para poder obtener la misma sensación que se tiene cuando se corre descalzo.

En un boletín de prensa, el gerente de Nike México, Luis Godínez, afirmó que se viajó hasta Chihuahua para que los rarámuris se probaran los tenis. También comentó que el deporte de correr es una forma natural en la etnia tarahumara, de ahí que sean considerados como una fuente de inspiración para los atletas.

Cómo mejorar la responsabilidad del diseñador en el uso de los referentes étnicos
La falta de apoyo legal de los diseños étnicos mexicanos es evidente. Del mismo modo, la diversidad de diseñadores que se inspiran en su iconografía y simbología para reflejarla en sus productos de diseño sin dar ningún crédito. Por ello, es necesario saber el uso lícito y responsable de estos diseños al inspirarse en su representación de identidad cultural. Es preciso entender el contexto de cada grupo étnico, y no solo emplear sus recursos como una copia para beneficiarse de estos, sin tomar en cuenta el trabajo de los artesanos de cada región.

De acuerdo con Marré (2012) la propiedad intelectual en el diseño de indumentaria a diferencia de otras industrias culturales como la música y el cine, no cuenta con el apoyo para el reconocimiento en la creación de este tipo de diseño. Menciona que un diseñador puede proteger su marca, pero son pocos los casos de aquellos que pueden patentar sus diseños. Cada diseñador podría registrar un dibujo, un nuevo tipo de tela, una textura, una forma, pero en la mayoría de los diseños iconográficos en las prendas no pueden ser patentados y no cuentan con derechos de autor. Debido a que son considerados productos de utilidad, es decir, que tienen la finalidad de vestir.

La habilidad que conlleva el diseño aunados los recursos de internet, muchas personas pueden tomar elementos iconográficos de alguna prenda u objeto e integrarlo a su propio diseño. En la industria del diseño de moda, la creatividad es infinita, ya que no hay limitaciones en el proceso creativo que inspire un diseño. Marré (2012), aclara que las creaciones y los estilos de los diseñadores, se vuelven bienes de uso público a partir de que salen a la venta. A partir de los cuales se puede inspirar para crear nuevos diseños o incluso solo copiarlos. Propiciando con ello una mayor competencia y agilización de los procesos creativos al obligar a los diseñadores a innovar constantemente. Se destaca que es la creatividad y la originalidad el fundamento para competir en la industria de la moda. Estas características generadas por la propia cultura, juegan un rol primordial al contribuir de manera significativa en la confección de prendas creativas. Los diseñadores de moda se forman con la herencia del patrimonio cultural tangible e 
intangible. La forma tangible es determinada por materiales, modelos, diseños, telas, entre otros. El patrimonio intangible es aquel dado por la reputación, la creatividad, los usos, las representaciones, las expresiones, los conocimientos y las técnicas (ídem).

La cultura de acuerdo con Barrère y Delabruyère (2008) se gesta a través de la herencia y la creatividad. Producto de esta es el diseño que se desarrolla con base en el patrimonio cultural. Cuando las industrias culturales parten de un conocimiento e ideas creativas, fomentan nuevos actos creativos.

Métodos La investigación de este trabajo fue de corte cualitativo. Dado que, posibilitó varias interpretaciones de la realidad y de los datos a través de las aportaciones de los estudiantes de nivel avanzado del Programa Educativo de Diseño Gráfico de la Universidad Autónoma de Ciudad Juárez (UACJ). En un primer acercamiento al grupo de estudio, se emplearon los siguientes instrumentos: encuestas, observación y entrevistas informales. Estos fueron empleados para saber acerca de su percepción, valoración o evaluación en materia de: registro de información y responsabilidad en el uso de diseños étnicos. La realización de este estudio estimó aproximadamente dieciocho meses.

Otro momento del estudio fue la investigación de fuentes bibliográficas, revistas, videos y ponencias. Posteriormente, se efectuó la recopilación de datos a través de una investigación de campo en: el Fomento Cultural de la Ciudad de México; el Instituto Mexicano de la Propiedad Industrial (IMPI) y la Comisión Nacional para el Desarrollo de los Pueblos Indígenas (CDI). Se emplearon fichas de recaudación de datos, fotografías y entrevistas semiformales.

Estos instrumentos dieron a conocer lo que estudiantes, inclusive docentes, consideraban sobre el valor o el reconocimiento de la propiedad intelectual. A partir de esto, se planteó la siguiente solución gráfica orientada a esta población estudiantil: un código de ética respaldado en la investigación desarrollada acerca de la responsabilidad social en el uso adecuado de diseños étnicos. Así como de, la creación de un colegio, comunidad o consejo de honor y justicia regulador de su cumplimiento.

Esta propuesta tiene como objetivo fomentar en los estudiantes una actitud ética y responsable en el uso de toda creación que inspireo base su trabajo. De manera que, sean capaces de retomar para crear una reconceptualización de diseño original. Otro objetivo es, despertar el interés por el conocimiento sobre los medios para el registro de la propiedad intelectual que todo diseñador profesional debe saber para la protección de sus diseños. Una vez delimitadas las características de la población estudiantil de Diseño Centrado en el Usuario (DCU) se optó por el modelo de Jakob Nielsen (en Arenzana, 2016) para su elaboración. 
Los primeros resultados de la encuesta realizada a los alumnos de nivel avanzado del Instituto de Arquitectura Diseño y Arte de la Universidad Autónoma de Ciudad Juárez, arrojó que estos tienen poco conocimiento en materia de la protección legal y el registro de la propiedad, al igual que con los conceptos de la propiedad intelectual, la propiedad industrial y los derechos de autor. La mayoría de los encuestados consideraron importante saber de los procedimientos para el registro del diseño. Además, de mostrar interés sobre cómo utilizar los recursos gráficos de forma responsable, la actitud ética hacia el uso adecuado de la iconografía y diseños étnicos junto con la importancia de dar crédito y reconocimiento a los diseñadores en los cuales se han inspirado, (ver la figura 1):

\section{USO ADEECUADODE}

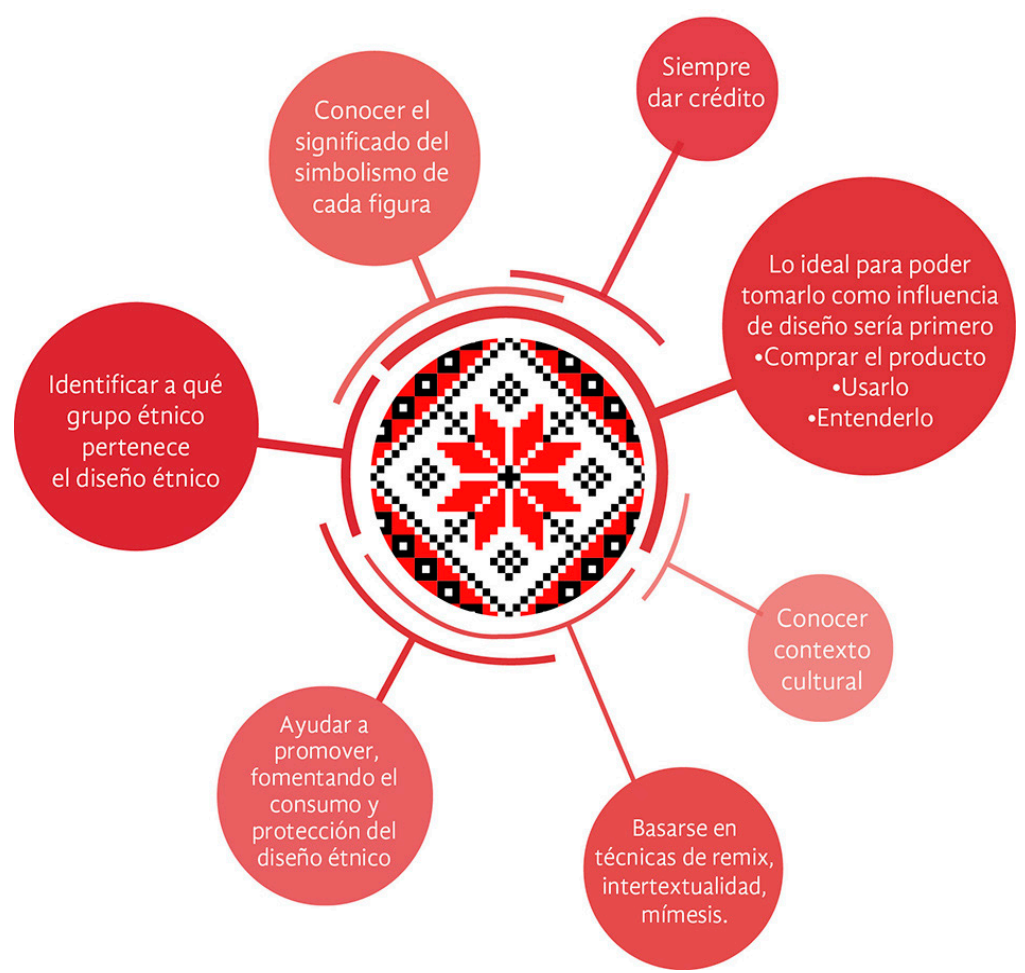

Figura 1. Uso adecuado de diseños étnicos. Fuente: Domínguez $(2017,129)$.

Se consideró con base en la cuestión del estado actual mencionada, la realización de un decálogo ético del diseñador de la comunicación visual. Para ello, fue necesaria la revisión de otros decálogos formulados por otros diseñadores u otras disciplinas del diseño que sirvan de antecedentes al proyecto. Como por ejemplo, el Decálogo del buen diseñador, de Dieter Rams, diseñador industrial alemán, autor conocido principalmente por ser la mente creativa detrás de la marca Braun (en Gubieda, 2015). Su frase más célebre es «Weniger, aber besser», que quiere decir «Menos, pero mejor». Dieter Rams formó este decálogo con los códigos fundamentales bajo los cuales confeccionar cada uno de los diseños de la empresa (ídem). 
Las normas son un fundamento del bien común. De manera que, resulta pertinente un decálogo para el diseñador en formación. Se partió para ello del Código ético del diseñador de la UNAM y el Decálogo de Rams, conjuntamente con mi experiencia como diseñadora gráfica. En la figura 2 se muestra el modelo de este decálogo diseñado por Domínguez (2017).

\section{DECÁLOGO ฮ DISEÑADOR}
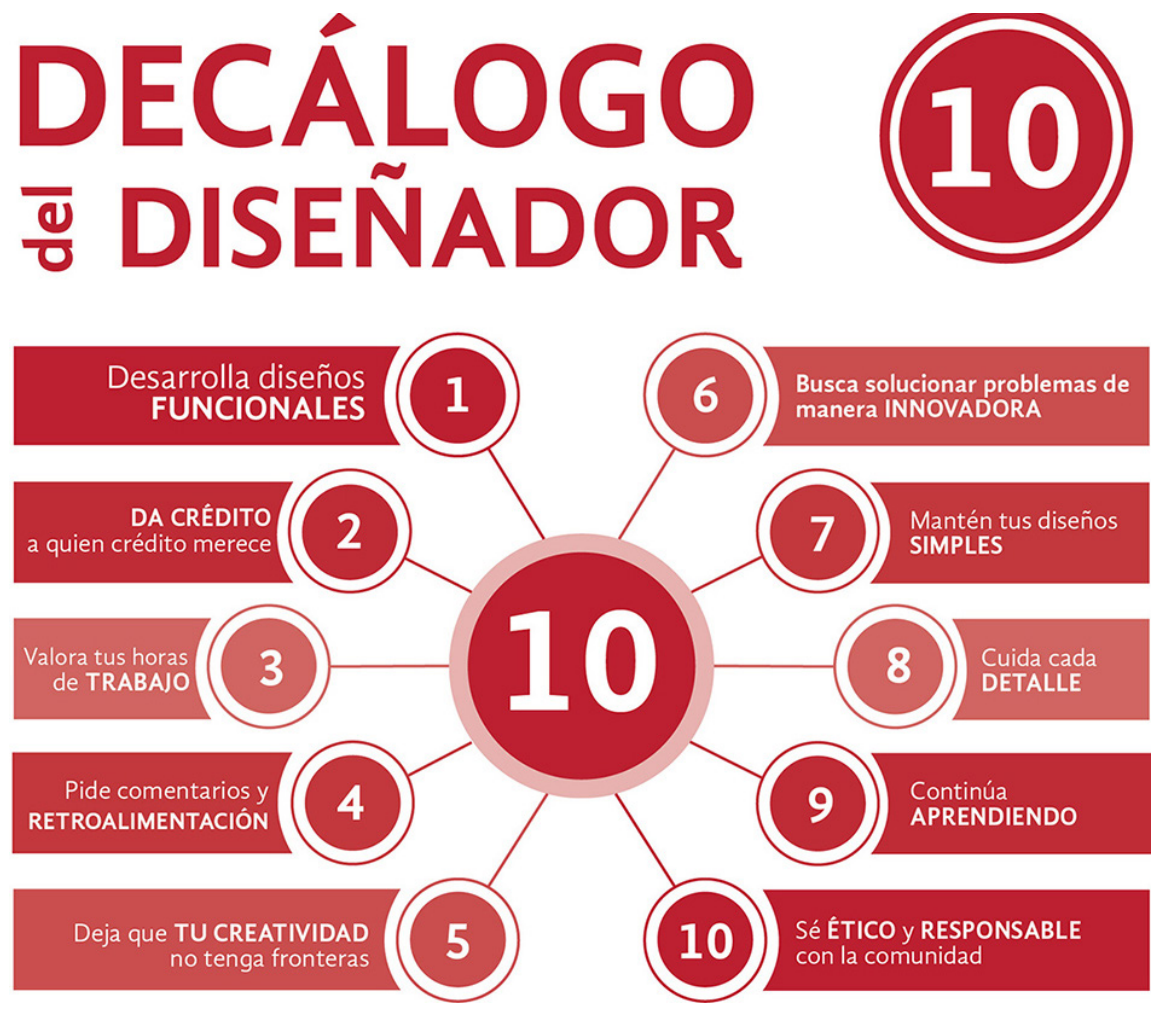

Figura 2. Decálogo del diseñador. Fuente: Domínguez (2017, 135).

La siguiente etapa de la investigación consistió en la realización de preguntas con relación en estos diez principios, cuyo propósito era saber si la información proporcionada fue pertinente a la problemática existente respecto a la falta de protección legal de la iconografía étnica mexicana y sus diseños. Fomentar mediante esto, la responsabilidad ética del diseñador en el uso de iconos étnicos (ver figuras 3 y 4). Estas preguntas se aplicaron a un grupo de estudio del cual el $50 \%$ era de diseñadores gráficos de nivel avanzado y, el $50 \%$ restante era el de usuarios externos en materia de diseño o que no es de su interés. Los resultados de verificación de la propuesta señala que la página web fue la mejor diseñada, lo cual, facilita la comprensión del tema principal, volviéndose un tema de su interés. Es importante mencionar que tanto diseñadores como usuarios externos comentaron sobre la usabilidad y la sencillez en el manejo de la información; principalmente que, entendieron la finalidad y el propósito inicial de esta investigación, para promover en cada uno de ellos la responsabilidad y ética al hacer uso de diseños étnicos. 


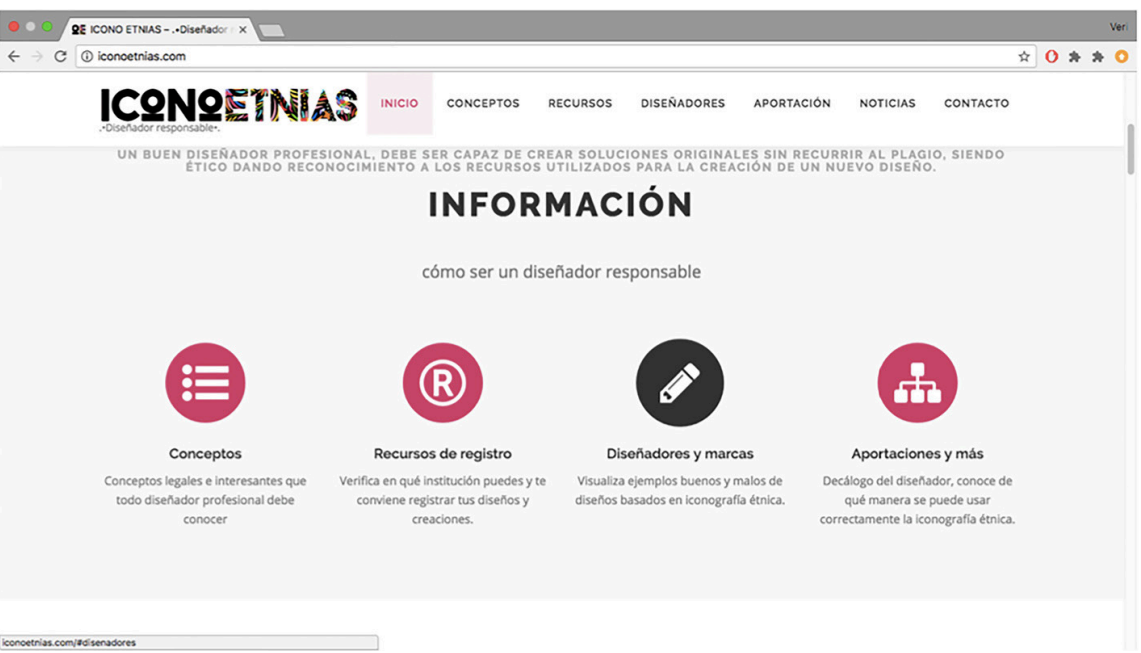

Figura 3. Página web iconoetnias.com. Fuente: Domínguez $(2017,177)$.

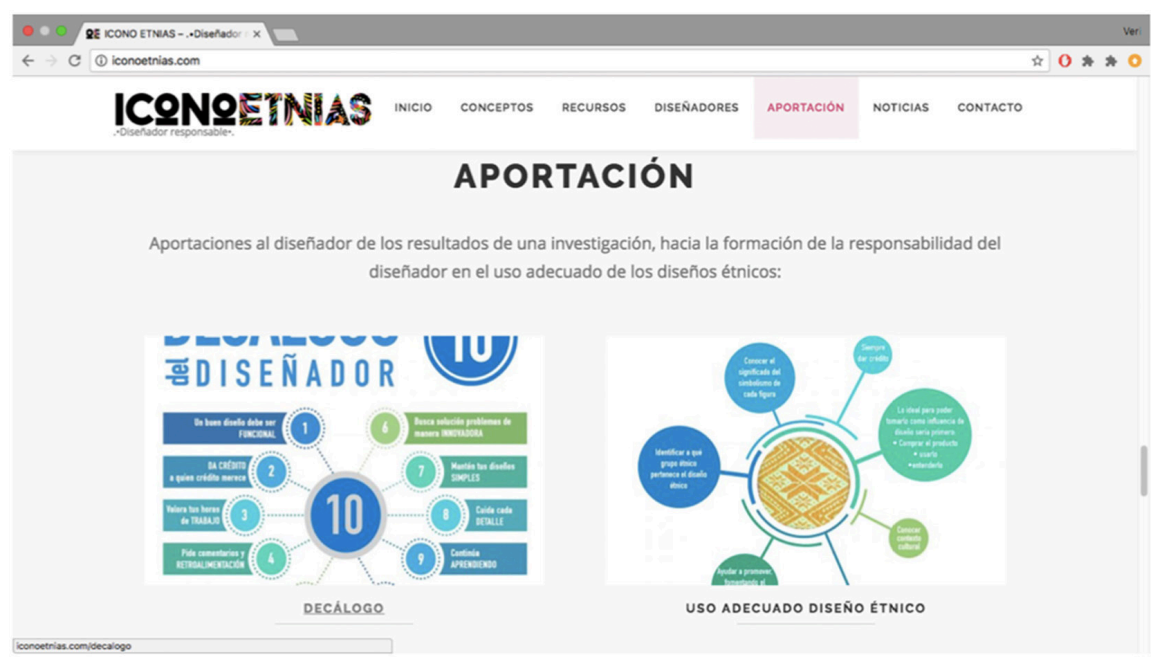

Figura 4. Página web iconoetnias.com, sección aportación. Fuente: Domínguez (2017, 180).

Conclusiones A manera de conclusión, es importante propiciar el debate sobre la ética del diseñador, la creatividad y la constante innovación; características sobresalientes que deben formar parte de todo diseñador. El ejercicio de reinventarse continuamente será su principal ventaja competitiva. Consecuente a ello, el valor percibido por el consumidor no solo será el de un diseño adquirido, sino el de una imagen y un concepto desarrollado. Incluso, dentro de lo que un diseñador serio debe considerar es la de adoptar una actitud responsable y ético al enfrentar los problemas complejos, lo cual propicia a generar mejores propuestas.

Usualmente los diseñadores quieren dar a conocer y representar su identidad cultural a través de sus diseños, cada diseñador cuenta con 
su propio estilo. Pero el proceso creativo que esté basado o inspirado en el trabajo de otros diseñadores, es necesario citar y brindar el debido reconocimiento y crédito autorial. Mismo principio que se aplica ante la creación que no cuenta con un registro legal, como es el caso del patrimonio perteneciente a los grupos étnicos, los cuales, hasta la fecha, no cuentan con un respaldo legal en el diseño iconográfico de sus artesanías.

Cabe hacer una mención respecto a la propuesta de ley aprobada en Guatemala para la protección de sus diseños étnicos, para ello se recurre a la reflexión del antropólogo Jesús Rafael Santaella, quien dijo vía telefónica a Mulato (2017) que: «en México no ha existido una propuesta de ley como esa». Mencionó también que para eso primeramente:

Debe haber interés por parte de autoridades en este tema. También se debe iniciar campañas de concientización en las comunidades explicando a las mujeres artesanas la situación sobre los plagios, así ellas pueden proponer iniciativas como la de las tejedoras en Guatemala [...] con una ley de propiedad intelectual colectiva se evitarían muchos plagios y las autoridades tendrían la obligación de responder a las denuncias (ídem).

Finalmente, los diseñadores de cualquier disciplina deben tomar conciencia de ser innovadores y multidisciplinarios, formar criterios con base en las distintas disciplinas, resolver problemas de comunicación visual dando el debido reconocimiento a quienes corresponda la autoría de cada diseño. La importancia de mantenerse informado en cuanto a las normas y los derechos de autor para su propio beneficio. Recalcando lo fundamental que es dar crédito y reconocimiento a quien es el legítimo autor. Es por ello que, a través de este trabajo se espera contribuir en la formación profesional diseñadores mexicanos que utilicen el esquema y decálogo de apoyo en esta materia.

\section{Referencias}

Arenzana, D. (2016). Principios de usabilidad web de Jackob Nielsen, Diseño UX [en línea]. Recuperado de https://es.semrush.com/blog/usabilidad -web-principios-jackob-nielsen/

Barrère, C. y Delabruyère, S. (2008). Intellectual Property Rights on Creativity and Heritage: the Case of Fashion Industry. Francia. Recuperado de http:// www.dime-eu.org/files/active/0/BarrerePAPER.pdf

Basail, A. (2006). Sociedad de hoy. Ensayos de sociología joven. La Habana: Ciencias Sociales.

Domínguez, V. (2017). El desamparo de la ley en el uso de diseños étnicos mexicanos: hacia una formación del diseñador responsable (tesis de maestría). Chihuahua: Universidad Autónoma de Ciudad juárez. 
Escobar, A. (2015). Acusan a diseñadora francesa de plagio a comunidad Mixe. Milenio, sección: Tendencias [en línea]. Recuperado de http://www.mi lenio.com/tendencias/Mixe_Tejidolsabel_MarantNeiman_Marcus-Susa na_HarpSanta_Maria_Tlahuitoltepec_0_522547950.html

Esquire. (2013). Nike y su inspiración Tarahumara [en línea]. Recuperado de http://www.esquirelat.com/moda/13/08/22/nike-y-inspiracion-tar ahumara/

Fletcher, K. (2010). Sustainable design in fashion \& textiles. Design journeys [en línea]. Recuperado de http://vistelacalle.com/27290/\%C2\%BF quien-es-kate-fletcher/

Fomento Cultural Banamex. (2016). Moda Siglo XX. Diseñadora Russek [en línea]. Recuperado de http://fomentoculturalbanamex.org/?s=russek

Fomento Cultural Banamex. (2016). Y el caso del «plagio» de la blusa de Oaxaca [en línea]. Recuperado de http://www.fomentoculturalbanamex.org/ gmap/el-caso-del-plagio-de-la-blusa-de-oaxaca/

González, C. (2013). Design Decalogue. Decálogo del diseñador [en línea]. Recuperado de https://www.domestika.org/es/projects/94038-design-de calogue-decalogo-del-disenador

Gubieda, P. (Noviembre, 2015). El tornillo que te falta. El decálogo del buen diseño, según Dieter Rams [en línea]. Recuperado de https://eltornillo quetefalta.net/2015/11/21/el-decalogo-del-buen-diseno-segun-die ter-rams/

Huidobro, S. (2005). Manual de Ética para el Diseño. Viña del Mar: Duoc UC.

López, C. (2013). Relación entre el diseño gráfico y las leyes. Conceptos y procesos. Puebla: Universidad de las Américas de Puebla.

López, L. (2016). La responsabilidad del diseñador como nuevo consumidor [en línea]. Montevideo. Recuperado de https://foroalfa.org/articulos/eleslabon-perdido-en-el-sistema-de-la-moda

Marré, S. (2012). La propiedad intelectual y el diseño de indumentaria de autor. Cuadernos del Centro de Estudios de Diseño y Comunicación. 42, 43-56.

Mendoza, P. (2012). Vestimenta como medio de representación de identidad sociocultural. Indumentaria y Moda, sección: La moda indígena [en línea]. Recuperado de http://fomentoculturalbanamex.org/moda/

Mulato, A. (2017). Un grupo de artesanas guatemaltecas propone una ley para detener el plagio de sus diseños. El país, sección: Verne [en línea]. Recuperado de http://verne.elpais.com/verne/2017/04/03/mexi co/1491194702_234171.html 
Torres, P. (2014). La moda y sus implicaciones [en línea]. Recuperado de ht tps://www.monografias.com/trabajos93/moda-y-sus-implicaciones/ moda-y-sus-implicaciones.shtml\#lavestimea

Universidad Nacional Autónoma de México, (2010). Código ético del diseñador y comunicador visual. México: Universidad Nacional Autónoma de México.

Acerca del autor

Maestra en estudios y procesos creativos en arte y diseño y licenciada en Diseño Gráfico por la Universidad Autónoma de Ciudad Juárez (UACJ). Actualmente trabaja como diseñadora de creación de imagen y publicidad para la diseñadora de moda Elda Bustamante en Ciudad Juárez, Chihuahua. De igual manera, labora para la Iglesia Metodista Nuevo Pacto en Phoenix, Arizona. También se desempeña para Super Good Movers en El Paso, Texas. Así como para la agencia Marketing Mix que trabaja con diferentes marcas en varios eventos de Ciudad Juárez, Chihuahua.

\section{Erika Anastacia Rogel Villalba}

Doctora en Investigación por el Colegio de Chihuahua (COLECH), Ciudad Juárez, Chihuahua, México y candidato a Doctor: Research in Art and Design in Sheffield Hallam University (SHU) Sheffield, England. Cuenta con una maestría en Research in Art and Design in Sheffield Hallam University (SHU). Así como estudios de licenciatura en Diseño Gráfico por la Universidad Autónoma de Ciudad Juárez (UACJ).

Profesora de Tiempo Completo (PTC) desde 1996, con Perfil Deseable (PRODEP). Sus áreas de investigación son: Metodologías en el Diseño, Diseño contra el Crimen, Diseño Social, Diseño Sostenible. Es miembro del Sistema Nacional de Investigación (SNI). Así como, miembro del Cuerpo Académico en Consolidación: Diseño Usuario y Entorno. Es también, integrante del Núcleo Académico Básico del Doctorado en Diseño y de la Maestría en Estudios y Procesos Creativos de Arte y Diseño en la UAC). Está Certificada en el Modelo Pedagógico de la UACJ. Ha dirigido más de 100 proyectos de tesis con enfoque social en Pregrado y 11 tesis concluidas de Posgrado.

Jefa de Evaluación y Acreditación de la UACJ, hasta el mes de octubre de 2018. Miembro del Consejo Académico de la UACJ durante el período 2006-2018. Coordinadora de Apoyo al Desarrollo Académico en el Instituto de Arquitectura Diseño y Arte del 2006-2017. 